\title{
Chiral Separation of $\alpha$-hydroxy Acids by Capillary Electrochromatography Using the Cathodic Detergent CTAB as Mobile Phase Additive to Speed up Analysis Time
}

\author{
Stefan Mohr, ${ }^{\mathrm{a}, \mathrm{b}}$ Goran Sepic, ${ }^{\mathrm{c}}$ and Martin Gerhard Schmid ${ }^{\mathrm{a}, *}$ \\ ${ }^{a}$ Department of Pharmaceutical Chemistry, Institute of Pharmaceutical Sciences, Karl-Franzens-University Graz, \\ Humboldtstrasse 46, A-8010 Graz, Austria \\ ${ }^{\mathrm{b}}$ Research Center Pharmaceutical Engineering, Graz, Austria \\ ${ }^{\mathrm{c}}$ Faculty of Pharmacy and Biochemistry, University of Zagreb, A. Kovacica 1, HR-10000 Zagreb, Croatia
}

RECEIVED APRIL 19, 2011; REVISED JUNE 1, 2011; ACCEPTED JUNE 21, 2011

\begin{abstract}
In order to speed up the analysis time, working with negatively charged compounds in capillary electromigration techniques often requires to change direction of the EOF. Negatively charged compounds intend to migrate towards the anode and are normally retained by the counter migrating EOF. Therefore the positively charged detergent cetyltrimethylammoniumbromide (CTAB) was used as mobile phase additive for the chiral separation of $\alpha$-hydroxy acids in capillary electrochromatography (CEC). A $25 \%$ particle loaded Ristocetin A continuous bed (CB) was used as stationary phase to separate a set of mandelic acid derivatives. Using mandelic acid and 4-bromo-mandelic acid as model substances the effect of different CTAB concentrations in the mobile phase on the migration time and the resolution was tested. It could be shown that with an increasing concentration of the $\mathrm{CTAB}$ the migration time decreased. The addition of $\mathrm{CTAB}$ to the mobile phase is a serious alternative to copolymerizing an anionic charge providing substances such as diallyl dimethylammonium chloride (DADMAC) in CB technology. (doi: 10.5562/cca1902)
\end{abstract}

Keywords: capillary electrochromatography, CEC, alpha-Hydroxy acids, continuous bed, macrocyclic antibiotics

\section{INTRODUCTION}

Because of its ubiquitous building blocks, L-amino acids and D-carbohydrates, the human organism represents an asymmetric environment in which active pharmaceutical ingredients (API) are showing effects. Therefore chiral drug substances show different interactions with body-proteins such as receptors or transport-molecules which may result in a restriction of the desired pharmacological effects to one of the enantiomers (eutomer). The unwanted enantiomer (distomer) may show undesirable side effects or even toxic effects. It represents at least an unnecessary burden for the organism. About half of drug substances are chiral but only $35 \%$ out of them are administered as pure enantiomers. ${ }^{1}$ With respect to this fact, in pharmaceutical industries it is more and more common to check the enantiomers of a promising racemic substance separately for their different physiological effects. Thus, the development of enantiomer separation techniques has attracted great attention in the past decades.
The development of chiral separation techniques is not only important for the characterization of APIs. The natural occurring chiral alpha hydroxy acids are widely used as building blocks for chiral drug substances. ${ }^{2}$ Because of their function as key metabolites in prokaryotic and eukaryotic cells they are used to determine metabolic states and physiological disorders as well. ${ }^{3-7}$ An increase of the D-lactic acid level in blood for example may give information about a bacterial infection. Other $\alpha$-hydroxy acids such as mandelic acid are used in skin peels fort the treatment of acne vulgaris. ${ }^{8}$

The afore mentioned applications justify the need of a fast, selective and easy to prepare instrumental method for the chiral determination of $\alpha$-hydroxy acids.

In literature several chromatographic and electrophoretic separation principles for the chiral determination of $\alpha$-hydroxy acids have been published. Using GC a preliminary derivatization step has to be performed. ${ }^{5}$ The use of different CSP e.g. macrocyclic antibiotics or immobilized antibodies in HPLC coupled to MS or UV detection is reported. ${ }^{7,9}$ Alternatively to those well-

\footnotetext{
* Author to whom correspondence should be addressed. (E-mail: martin.schmid@uni-graz.at)
} 
Table 1. Chiral separation of $\alpha$-hydroxy acids in CEC on a $25 \%$ particle loaded Ristocetin A CB without CTAB as mobile phase additive.

\begin{tabular}{lcccc}
\hline Sample & $t_{1} / \min$ & $t_{2} / \min$ & $t_{2}: t_{1}$ & $R_{\mathrm{s}}$ \\
\hline Mandelic acid & 18.21 & 32.05 & 1.76 & 2.92 \\
4-Bromomandelic acid & 18.45 & 25.41 & 1.37 & 1.96 \\
p-Methoxymandelicacid & 19.09 & 34.68 & 1.82 & 3.03 \\
p-Hydroxymandelic acid & 25.33 & 39.75 & 1.57 & 2.16 \\
3-Hydroxymandelic acid & 27.27 & 39.23 & 1.44 & 1.63 \\
3-Hydroxy-4-methoxymandelic acid & 38.03 & 49.89 & 1.31 & 1.22 \\
\hline
\end{tabular}

Conditions: $25 \%$ particle loaded Ristocetin A CB (36 cm); mobile phase, $0.05 \%$ TEA and $0.1 \%$ acetic acid in methanol; voltage, $20 \mathrm{kV}$ to the anode; applied pressure, 12 bar at inlet and outlet; injection: $6 \mathrm{bar} \times 6 \mathrm{sec}$.

established techniques, capillary electrophoresis and capillary electrochromatography turned out to produce good separation results using different types of chiral selectors. ${ }^{4,10-12}$

CEC is nowadays a wide spread technique to perform chiral separations. In CEC there are different possibilities to immobilize the chiral selector in the column. The simplest way is to adsorb or chemically bond the chiral selector to the inner wall of the capillary. It may happen that the selector concentration is not sufficient to achieve satisfying chiral separation in those open tubular CEC systems. To improve resolution packed capillaries are used. The packing of capillaries with a chiral stationary phase bonded to silica requires the preparation of limiting frits by sintering a zone of the silica packing. To circumvent this complicated procedure monolithic stationary phases turned out to be a good alternative in recent years. Silica based monolithic phases are often prepared by a solgel process employing polycondensation of alkoxysilanes. $^{13}$ In situ polymerization of organic monomers including chiral selectors directly in the capillary leads to polymeric monoliths which are inexpensive and easy to prepare. ${ }^{14,15} \mathrm{Schmid}$ et al. combined polymeric monoliths with commercially available chiral selectors chemically bonded to silica particles. ${ }^{16}$ A monomer solution with suspended silica particles was drawn into a capillary and was polymerized. Good chiral separations of $\alpha$-hydroxy acids were shown by using L-4-hydroxyproline ${ }^{11}$ bonded to $3 \mu \mathrm{m}$ silica material in ligand-exchange CEC and by using the macrocyclic antibiotics Ristocetin $\mathrm{A}$ and teicoplanin aglycone (TAG) bonded to $3 \mu \mathrm{m}$ silica material. $^{16}$

Macrocyclic antibiotics of the glycopeptide type were first introduced by Armstrong et al. ${ }^{17}$ and are nowadays wide spread chiral selectors in HPLC, CE and CEC.

The EOF plays an important role on the separation time of $\alpha$-hydroxy acids. With silica based CSPs a cathodic EOF is generated because of the remaining free silanol groups on the silica particles. An increase in analysis time and peak broadening is observed be- cause the negatively charged $\alpha$-hydroxy acids are migrating to the anode and are thereby strongly retained by the EOF. To speed up the analysis time Schmid et al. copolymerized the positive charge providing reagent DADMAC in the afore mentioned experiments. ${ }^{16}$ With this method good enantioseparations in a relatively short time were achieved for $\alpha$-hydroxy acids in LE-CEC both with Ristocetin A and with TAG stationary phase.

This article deals with the use of the cationic detergent $\mathrm{CTAB}$ as mobile phase additive in CEC to reverse the EOF. With this method it was possible to decrease the separation time of a series mandelic acid derivatives. A particle loaded continuous bed with Ristocetin A chemically bonded to $3 \mu \mathrm{m}$ silica gel was used as stationary phase without any copolymerized charge providing reagent. CTAB was simply added to the mobile phase and showed a marked accelerating effect on the retention times. The CTAB is adsorbed to negatively charged silanolic groups and can easily be removed by flushing the capillary with pure acetonitrile. Thus it is possible to use the same capillary for the enantioseparation of other chiral substances e.g. amino acids.

\section{EXPERIMENTAL}

\section{Instrumentation}

A PrinCE capillary electrophoresis system (PrinCE Technologies, Emmen, Netherlands) equipped with a LAMBDA 1100 UV/VIS detector (Bischoff Analysentechnik, Leonberg, Germany) and an external pressure device (12 bar), was used for the CEC measurements. Detection was performed via on column measurement of the UV absorption at $208 \mathrm{~nm}$. Operation temperature was set to $25^{\circ} \mathrm{C}$. Instrument controlling and data processing was carried out by DAX software (6.0) installed on a personal computer.

Measurements were accomplished in $200 \mu \mathrm{m}$ ID fused silica capillaries (Micro Quartz, Munich, Germany) with a total length of $70 \mathrm{~cm}$. The effective length containing the particle loaded CB was $36 \mathrm{~cm}$. A voltage 
Table 2. Influence of different concentrations of $\mathrm{CTAB}$ in the mobile phase on the enantioseparation of mandelic acid and 4-bromomandelic acid in CEC on a $25 \%$ particle loaded Ristocetin A CB.

\begin{tabular}{|c|c|c|c|c|c|c|c|c|c|c|}
\hline \multirow[b]{2}{*}{$c(\mathrm{CTAB}) / \mathrm{mmol} \mathrm{dm}^{-3}$} & \multicolumn{5}{|c|}{ Mandelic Acid } & \multicolumn{5}{|c|}{ 4-bromomandelic Acid } \\
\hline & 0 & $0^{(\mathrm{a})}$ & 10 & 20 & 25 & 0 & $0^{(\mathrm{a})}$ & 10 & 20 & 25 \\
\hline$t_{1} / \min$ & 18.21 & 17.17 & 12.22 & 12.03 & 11.15 & 18.45 & 19.89 & 12.43 & 11.05 & 10.64 \\
\hline$t_{2} / \min$ & 32.05 & 31.45 & 18.17 & 19.10 & 17.71 & 25.41 & 25.27 & 15.33 & 14.13 & 13.56 \\
\hline$t_{2}: t_{1}$ & 1.76 & 1.83 & 1.49 & 1.59 & 1.59 & 1.38 & 1.27 & 1.23 & 1.28 & 1.27 \\
\hline$R_{\mathrm{s}}$ & 2.92 & 3.05 & 2.73 & 2.10 & 1.64 & 1.96 & 1.16 & 1.61 & 0.78 & 1.08 \\
\hline
\end{tabular}

Conditions: $25 \%$ particle loaded Ristocetin A CB $(36 \mathrm{~cm})$; mobile phase, $0.05 \%$ TEA and $0.1 \%$ acetic acid in methanol containing different concentrations of $\mathrm{CTAB}$; voltage, $20 \mathrm{kV}$ to the anode; applied pressure, 12 bar at inlet and outlet; injection: 6 bar $\times 6 \mathrm{sec}$.

(a) after removing the adsorbed CTAB by flushing the capillary with pure acetonitrile.

of $20 \mathrm{kV}$ was applied during the measurements. Because of the negatively charged analytes and the reversed EOF measurements were performed in negative mode. To prevent air bubble formation a pressure of 12 bar was applied at both ends during the runs.

\section{Chemicals and Solutions}

All chemicals were of analytical grade. Acetic acid, triethylamine, methanol and $\mathrm{N}$-cetyltrimethylammoniumbromide were obtained from VWR International. Water was deionized and doubly distilled. Piperazine diacrylamide, ammonium peroxodisulfate, and $N, N, N^{\prime}, N^{\prime}-$ tetramethylethylenediamine (TEMED) were purchased from Bio-Rad Laboratories (Hercules, CA, USA). Methacrylamide, $\quad \gamma$-methacryloxypropyltrimethoxysilane and all tested $\alpha$-hydroxy acids were from Sigma-Aldrich Chemicals (St.Louis, MO, USA). Ristocetin A chemically bonded to $3 \mu \mathrm{m}$ silica gel was a gift from Astec (Whippany, NJ, USA).

The mobile phase was prepared by dissolving a different amount of CTAB in a solution of $0.05 \%$ TEA and $0.1 \%$ acetic acid in methanol. All mobile phases were degassed for 10 min with helium 5.0 and filtered through a $0.2 \mu \mathrm{m}$ filter. Samples were dissolved in methanol $\left(1-3 \mathrm{mg} \mathrm{cm}^{-3}\right)$ and injected hydrodynamically or electrokinetically.

\section{Preparation of the Particle-loaded Continuous Bed}

To introduce a double-bond function at the capillary inner wall the $200 \mu \mathrm{m}$ ID fused silica capillaries were silanized with $\gamma$-methacryloxypropyltrimethoxy-silane after preconditioning the capillary with acetone $0.1 \mathrm{M}$ hydrochloric acid and $0.1 \mathrm{M}$ sodium hydroxide solution.

The preparation of the monolith was performed following the method of Schmid et al. ${ }^{16} \mathrm{~A}$ defined monomer solution containing $25 \%$ silica gel was drawn into the capillary till the detection window and polymerization was started by addition of ammonium peroxodisulfate and TEMED. To obtain an uncharged $\mathrm{CB}$ the charge providing DADMAC or vinylsulfonic acid was not added.
After some hours the polymerization was completed and the capillary was cut to the desired length. Before using it the capillary was flushed with water and mobile phase.

\section{RESULTS AND DISCUSSION}

The effect of different concentrations of CTAB in the mobile phase on the retention time and resolution of the two enantiomers of mandelic acid derivatives was examined. Without any CTAB in the mobile phase a set of 6 substances was tested as it is shown in Table 1. Without any charge providing reagent the hydroxy acids are migrating against a weak EOF which is directed to the cathode and results from the free silanol groups on the capillary inner wall and the silica particles. Therefore the $\alpha$-hydroxy acids are retained. All 9 substances could be resolved under this conditions within $55 \mathrm{~min}$.

Mandelic acid and 4-bromomandelic acid were chosen as model substances to study the effect of different $\mathrm{CTAB}$ concentrations on retention time and resolution (Table 2).

As expected retention times are decreasing with increasing CTAB concentrations. The effect of speeding up is attributable to the accumulation by ion-ion interaction of the positively charged ammonium group of the CTAB molecule and negatively charged silanol groups. If all negatively charged groups are saturated the excess of CTAB is adsorbed to the hydrophobic chain of the already adsorbed $\mathrm{CTAB}$ and therefore builds a positively charged surface is created which allows to generate an EOF directed to the anode. The fastest separations were obtained at a $\mathrm{CTAB}$ concentration of $25 \mathrm{mM}$. Figure 1 shows the resolution of mandelic acid without $\mathrm{CTAB}$ in the mobile phase with $25 \mathrm{mM}$ CTAB in the mobile phase and after removing the CTAB by washing the capillary with pure acetonitrile. For mandelic acid resolution ranged from 2.73 to 4.82 and for 4-bromomandelic acid from 1.61 to 3.47 with different $\mathrm{CTAB}$ concentrations. The cathodic detergent was removed successfully from the capillary by flushing with pure 


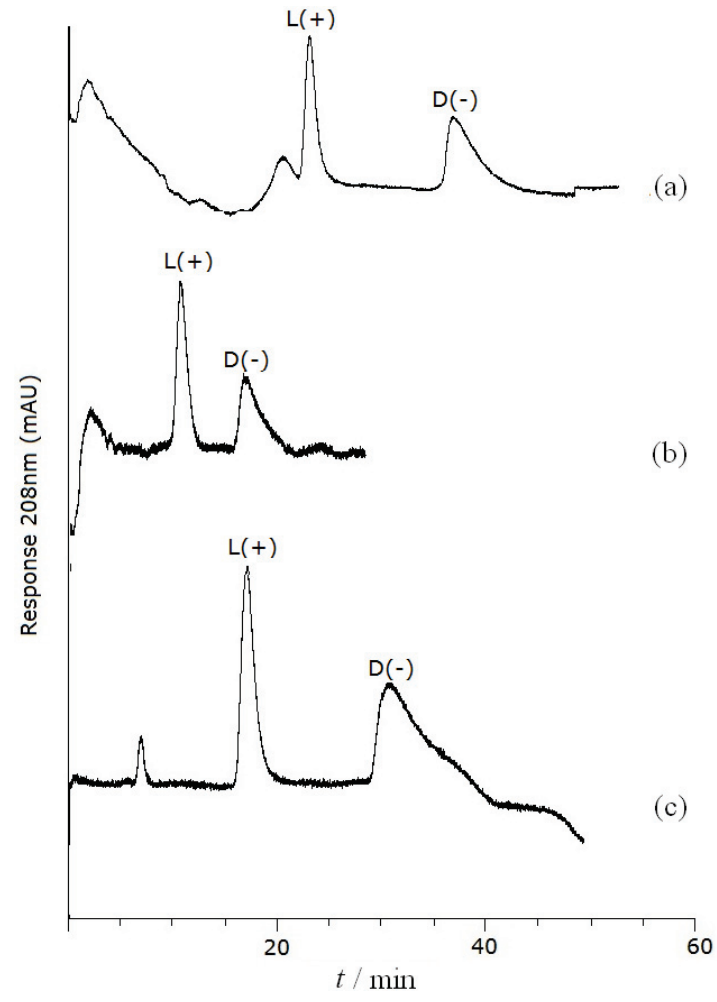

Figure 1. Comparison of the enantioseparation of mandelic acid (a) without $\mathrm{CTAB}$ as mobile phase additive, (b) with 25 mM CTAB, (c) after removing CTAB by washing the capillary with pure acetonitrile. Conditions: $25 \%$ particle loaded Ristocetin A CB (36 cm); mobile phase, $0.05 \%$ TEA and $0.1 \%$ acetic acid in methanol containing different concentrations of CTAB; voltage, $20 \mathrm{kV}$ to the anode; applied pressure, 12 bar at inlet and outlet; injection: 6 bar $\times 6$ sec.

acetonitrile for about 2 hours. After flushing retention times were similar to those at the start conditions. Therefore the same capillary was usable for the fast separation of $\alpha$-hydroxy acids and e.g. positively charged compounds.

\section{CONCLUSION}

It was shown that the cathodic detergent CTAB is suitable as mobile phase additive to speed up the separation of the negatively charged $\alpha$-hydroxy acids. As stationary phase a $25 \%$ particle loaded Ristocetin A CB was used. At the beginning a set of $9 \alpha$-hydroxy acids was analysed using a mobile phase without any CTAB. The model substances mandelic acid and 4-bromo mandelic acid were used to test the effect of different CTAB concentrations in the mobile phase by means of retention time and resolution. The shortest retention times were obtained at a concentration of $25 \mathrm{mM} \mathrm{CTAB}$ in the mobile phase. After flushing the capillary with pure acetonitrile the capillary showed nearly the same behaviour than before CTAB was used. It was shown that it is not urgently needed to copolymerize anionic charge providing substances such as DADMAC to reverse the EOF for the analysis of negatively charged compounds by using particle loaded CBs as stationary phases.

\section{REFERENCES}

1. J. S. Millership and A. Fitzpatrick, Chirality 5 (1993) 573-576.

2. G. M. Coppola, H. F. Schuster, Alpha-Hydroxy Acids in Enantioselective Synthesis, Wiley-VCH, Weinheim, 1997.

3. E. J. Franco, H. Hofstetter, and O. Hofstetter, J. Pharm. Biomed. Anal. 49 (2009) 1088-1091.

4. S. Fanali, P. Catarcini, C. Presutti, M. G. Quaglia, and P. G. Righetti, Electrophoresis 24 (2003) 904-912.

5. Y. Inoue, T. Shinka, M. Ohse, M. Kohno, K. Konuma, H. Ikawa, and T. Kuhara, J. Chromatogr. B 855 (2007) 109-114.

6. B. Y. Lee and T. F. Thurmon, Clin. Chim. Acta 218 (1993) 215-222.

7. E. J. Franco, H. Hofstetter, and O. Hofstetter, J. Pharm. Biomed. Anal. 46 (2008) 907-913.

8. V. K. Garg, S. Sinha, and R. Sarkar, Dermatol. Surg. 35 (2009) 59-65.

9. D. Norton, B. Crow, M. Bishop, K. Kovalcik, J. George, and J. A. Bralley, J. Chromatogr. B 850 (2007) 190-198.

10. L. Tan, Y. Wang, X. Liu, H. Ju, and J. Li, J. Chromatogr. B 814 (2005) 393-398.

11. M. G. Schmid, J. Koidl, P. Wank, G. Kargl, H. Zohrer, and G. Gübitz, J. Biochem. Biophys. Meth. 70 (2007) 77-85.

12. M. G. Schmid, O. Lecnik, U. Sitte, and G. Gübitz, J. Chromatogr. A 875 (2000) 307-314.

13. N. Ishizuka, H. Minakuchi, K. Nakanishi, N. Soga, H. Nagayama, K. Hosoya, and N. Tanaka, Anal. Chem. 72 (2000) 1275-1280.

14. B. Sellergren, J. Chromatogr. A 906 (2001) 227-252.

15. E. Turiel and A. Martin-Esteban, Anal. Bioanal. Chem. 378 (2004) 1876-1886.

16. M. G. Schmid, J. Koidl, C. Freigassner, S. Tahedl, L. Wojcik, T. Beesley, D. W. Armstrong, and G. Gübitz, Electrophoresis 25 (2004) 3195-3203.

17. D. W. Armstrong, Y. B. Tang, S. S. Chen, Y. W. Zhou, C. Bagwill, and J. R. Chen, Anal. Chem. 66 (1994) 1773-1784. 